\title{
Avaliação da ocorrência de doenças periodontais entre os pacientes atendidos em uma clínica escola de odontologia de um centro universitário do sudoeste da Bahia
}

\author{
Evaluation of the occurrence of periodontal diseases among patients seen at a dental school clinic of \\ a university center in southwestern Bahia
}

Evaluación de la ocurrencia de enfermedades periodontales en pacientes atendidos en la clínica de una escuela de odontología en un centro universitario en el suroeste de Bahía

\begin{abstract}
Resumo
O presente estudo tem como objetivo determinar a ocorrência da doença periodontal entre os pacientes atendidos na Faculdade Independente do Nordeste, identificando quais doenças periodontais são mais prevalentes e observando os possíveis fatores de risco a partir dos dados obtidos através da análise dos prontuários. Na pesquisa foram incluídos 141 prontuários de pacientes atendidos nessa mesma clínica escola, dos sexos feminino e masculino, com idades entre 22 a 73 anos. Os dados coletados foram tabelados em um banco de dados elaborado no Microsoft Excel (Microsoft Corporation, USA), onde foram tabulados o número de pacientes, sexo, idade, etnia, presença ou ausência de doença sistêmica relatada pelo paciente através da anamnese presente em seu prontuário, data do exame periodontal e diagnóstico periodontal. A análise estatística foi realizada através dos testes de Qui-quadrado, Mann-Whitney e Kruskal-Wallis. De acordo com os resultados logrados nesta pesquisa, observou-se que há alta ocorrência de doenças periodontais, comprovando a relação da doença com a condição sistêmica do paciente. Os dados avaliados evidenciaram um alto índice da ocorrência de doenças periodontais, identificando a mais prevalente, pontuando os fatores de risco e correlacionando-as com doenças sistêmicas.
\end{abstract}

Palavras-chave: Doenças periodontais; Biofilme; Gengivite; Periodontite; Higiene bucal.

\begin{abstract}
This study aims to determine the occurrence of periodontal disease among patients seen at the Faculdade Independente do Nordeste, identifying which periodontal diseases are more prevalent and observing the possible risk factors from the data obtained through the analysis of medical records. The research included 141 medical records of patients seen at the same school clinic, male and female, aged between 22 and 73 years. The collected data were tabulated in a database elaborated in Microsoft Excel (Microsoft Corporation, USA), where the number of patients, sex, age, ethnicity, presence or absence of systemic disease reported by the patient through the anamnesis present in their medical record, date of periodontal examination and periodontal diagnosis. Statistical analysis was performed using the Chi-square, Mann-Whitney and Kruskal-Wallis tests. According to the results obtained in this research, it was observed that there is a high occurrence of periodontal diseases, proving the relationship of the disease with the systemic condition of the patient. risk factors and correlating them with systemic diseases.
\end{abstract}

Keywords: Periodontal diseases; Biofilm; Gingivitis; Periodontitis; Oral hygiene.

\section{Resumen}

Este estudio tiene como objetivo determinar la ocurrencia de enfermedad periodontal entre pacientes atendidos en la Faculdade Independente do Nordeste, identificando qué enfermedades periodontales son más prevalentes y observando los posibles factores de riesgo a partir de los datos obtenidos mediante el análisis de historias clínicas. La investigación incluyó 141 historias clínicas de pacientes atendidos en la misma clínica escolar, hombres y mujeres, con edades comprendidas entre los 22 y los 73 años. Los datos recolectados fueron tabulados en una base de datos elaborada en Microsoft Excel (Microsoft Corporation, EE. UU.), Donde el número de pacientes, sexo, edad, etnia, presencia o ausencia de enfermedad sistémica reportada por el paciente a través de la anamnesis presente en su historia clínica, fecha del examen periodontal y diagnóstico periodontal. El análisis estadístico se realizó mediante las 
pruebas de Chi-cuadrado, Mann-Whitney y Kruskal-Wallis. De acuerdo con los resultados obtenidos en esta investigación, se observó que existe una alta ocurrencia de enfermedades periodontales, comprobando la relación de la enfermedad con la condición sistémica del paciente, factores de riesgo y correlacionándolos con enfermedades sistémicas.

Palabras clave: Enfermedades periodontales; Biofilm; Gingivitis; Periodontitis; Higiene bucal.

\section{Introdução}

A doença periodontal (DP) é caracterizada por um grupo de patologias de curso crônico, com etiologia multifatorial, podendo ocasionar diversos malefícios à saúde geral do indivíduo, por estar associada a outras doenças sistêmicas (Silva et al., 2020). Afetam cerca de 20 a 50\% da população mundial, sendo considerada a segunda maior causa de patologia dentária. Constitui-se, assim, um problema de saúde pública, predominante em adolescentes, adultos e indivíduos mais velhos (Nazir, 2017).

A etiologia da DP é compreendida pela inter-relação entre o agente, o hospedeiro e o meio ambiente. Nos últimos anos, foi demonstrado que a doença é resultado de complexas interações entre o biofilme bacteriano e a resposta imune do paciente, que levam à desregulação da resposta inflamatória (Kinane, Stathopoulou \& Papapanou, 2017). A sua progressão é determinada pelas características morfológicas dos tecidos afetados, o que a diferencia de outras doenças infecciosas e as manifestações clínicas estão diretamente ligadas às propriedades agressoras dos microrganismos e da capacidade do hospedeiro em resistir à agressão (Santos, Bittencourt, Terezan \& Rocha, 2020).

A DP manifesta-se inicialmente como uma gengivite, definida como uma inflamação superficial do tecido gengival, em que o epitélio de união se mantém unido ao dente, apesar das alterações patológicas, não havendo perda de inserção. Caso sejam removidos os fatores etiológicos, esta patologia pode se tornar reversível. No entanto, pode apresentar-se como um predecessor na perda de inserção dentária se os fatores etiológicos não forem eliminados (Miyamoto, Kumagai, Khan, \& Reddy, 2019; Slot, 2020).

As periodontites são compreendidas como uma inflamação que acarreta a destruição do periodonto e ocorre quando as alterações patológicas presentes na gengivite progridem até ocasionar uma destruição do ligamento periodontal e migração apical do epitélio de união. O acúmulo de biofilme bacteriano, ao nível dos tecidos mais profundos, causa perda de inserção por destruição do tecido conjuntivo e por reabsorção do osso alveolar (Miyamoto, et. al; 2019).

Os fatores predisponentes da DP compreendem a anatomia dental favorável à má higienização, higiene bucal traumática, acabamentos inadequados das restaurações, uso de aparelhos ortodônticos, acúmulo de biofilme bacteriano e presença de cálculo dental. A nível sistêmico podem ser listadas as doenças genéticas, sanguíneas, AIDS, gravidez (pela atuação de determinados hormônios), puberdade (onde os hábitos de higiene bucal são facilmente negligenciados), e tabagismo. Além desses fatores, a resposta imunoinflamatória do hospedeiro tem influência direta na formação das doenças periodontais (Rodrigues, Medeiros, Sousa, Sampaio \& Rodrigues, 2018).

Perante o que foi apresentado, sugere-se a realização de estudos regionais com o intuito de desenvolver pesquisas e programas de prevenção, diagnóstico e tratamento voltados para populações locais com o objetivo de atendê-los com mais eficiência e promover melhorias na qualidade da saúde, além de gerar a redução da incidência e da prevalência da doença periodontal. Dessa forma, o objetivo do presente estudo foi conhecer a prevalência das doenças periodontais entre os pacientes atendidos em uma Faculdade de odontologia Sudoeste da Bahia, identificando quais doenças periodontais são mais prevalentes e pontuar os possíveis fatores de riscos relatados durante a anamnese e descritos nos prontuários. 


\section{Metodologia}

Preconizado por Estrela, (2018), esta pesquisa trata-se de um estudo observacional retrospectivo desenvolvido em uma Clínica escola do curso de Odontologia de uma Faculdade do Sudoeste da Bahia, sendo este aprovado pelo Comitê de Ética e Pesquisa, segundo resolução vigente para Ética em Pesquisa em Seres Humanos n 466/12 do Conselho Nacional de Saúde (Ministério da Saúde, DF), além de autorização da Instituição para coleta de dados.

A pesquisa foi realizada na Secretaria da Clínica Escola de Odontologia de uma faculdade privada situada no sudoeste da Bahia. Foram analisados 285 prontuários de pacientes atendidos nessa mesma instituição nos períodos entre 2015 a 2021 , com faixa etária entre 22 a 73 anos. Após aplicados os critérios de seleção (prontuários preenchidos adequadamente, constando todas as informações pertinentes ao estudo, conferidos e assinados pelos professores responsáveis pelos atendimentos na clínica escola, tornando-os elegíveis), foram incluídos na amostra 141 prontuários elegíveis. Os demais foram excluídos pertinentes à falta de assinatura do professor responsável e/ou informações incompletas.

Para a realização do diagnóstico periodontal nessa clínica escola é preconizado o exame periodontal completo para todos os pacientes dentados, no qual é feito a sondagem com o uso de uma sonda periodontal milimetrada de Williams para verificar a profundidade de sondagem (PS) e o nível de inserção clínica (NIC). Além desses parâmetros, são avaliados o índice de sangramento gengival (IG) e o índice de biofilme (IB). Os critérios para o diagnóstico da DP são baseados nos critérios da Academia Americana de Periodontia (AAP, 2018). Considera-se periodontite os casos de pacientes que apresentam bolsas periodontais com profundidade de sondagem maior que três milímetros e perda de inserção clínica. Dentre os casos que não apresentarem perda de inserção, aqueles que apresentarem sangramento à sondagem em mais de 10\% dos sítios, receberão diagnóstico de gengivite, e quando o índice de sangramento foi inferior a esse valor, receberão a classificação de "saúde gengival”. Além da avaliação clínica é realizada uma anamnese detalhada de cada paciente para obtenção de informações que serão capazes de identificar possíveis fatores de risco que podem afetar o diagnóstico ou o processo de diagnóstico da doença.

Os dados coletados dos prontuários foram tabulados em um banco de dados elaborado no Microsoft Excel (Microsoft Corporation, USA), onde foram registrados o número de pacientes, sexo, idade, etnia, presença ou ausência de doença sistêmica relatada pelo paciente através da anamnese presente em seu prontuário, data do exame periodontal e diagnóstico periodontal.

A análises estatísticas foram realizadas por meio do software Statistical Package for the Social Sciences - SPSS, verão 22.2 para Windows. A análise da proporção absoluta em função sexo, diagnóstico periodontal e condições sistêmicas associadas foi realizada pelo teste do Qui-quadrado para uma amostra. As idades de mulheres e homens foram comparadas pelo teste de kruskal-Wallis, também utilizado para comparar as idades em relação ao diagnóstico e doenças sistêmicas. O nível de significância foi estabelecido em $5 \%$.

\section{Resultados}

No que se refere às características biosociodemográficas, a amostra composta por 141 indivíduos, apresentou média de idade de 36,79 ( \pm 10,29) anos, com predominância do sexo feminino 74 (52,5\%) e da raça leucoderma e melanoderma, ambas com 69 (48,9\%) dos casos. Em questões clínicas, $82(58,2 \%)$ dos participantes apresentaram alguma doença sistêmica, das quais hipertensão é a mais comum com 48 (34,0\%) das situações. Além disso, o perfil da amostra se mostrou majoritariamente não tabagista, afirmando não ser fumante em $96(68,1 \%)$ dos casos, conforme esboça a Tabela 1. 
Tabela 1. Características biosociodemográficas e clínicas da amostra.

\begin{tabular}{|c|c|c|}
\hline VARIÁVEIS & \multirow{2}{*}{\multicolumn{2}{|c|}{$\begin{array}{l}\text { média } \pm \mathbf{d p}^{1} \\
36,79 \pm 10,29\end{array}$}} \\
\hline Idade, anos & & \\
\hline SEXO & $\mathbf{n}$ & $\%$ \\
\hline Masculino & 67 & 47,5 \\
\hline Feminino & 74 & 52,5 \\
\hline \multicolumn{3}{|l|}{ RAÇA } \\
\hline Leucoderma & 69 & 48,9 \\
\hline Melanoderma & 69 & 48,9 \\
\hline Faioderma & 3 & 2,2 \\
\hline \multicolumn{3}{|l|}{ DOENÇA SISTÊMICA } \\
\hline Ausente & 59 & 41,8 \\
\hline Hipertensão & 48 & 34,0 \\
\hline Diabetes & 22 & 15,6 \\
\hline Problemas renais & 7 & 5,0 \\
\hline Anemia & 5 & 3,6 \\
\hline \multicolumn{3}{|l|}{ TABAGISMO } \\
\hline Não fumante & 96 & 68,1 \\
\hline Fumante & 36 & 25,5 \\
\hline Ex. fumante & 9 & 6,4 \\
\hline
\end{tabular}

Os dados contidos no Gráfico 1, mostram que a doença periodontal prevalente é gengivite 64 (45,4\%), seguida de periodontite com $59(41,8 \%)$ dos casos.

Gráfico 1. Diagnóstico de doença periodontal.

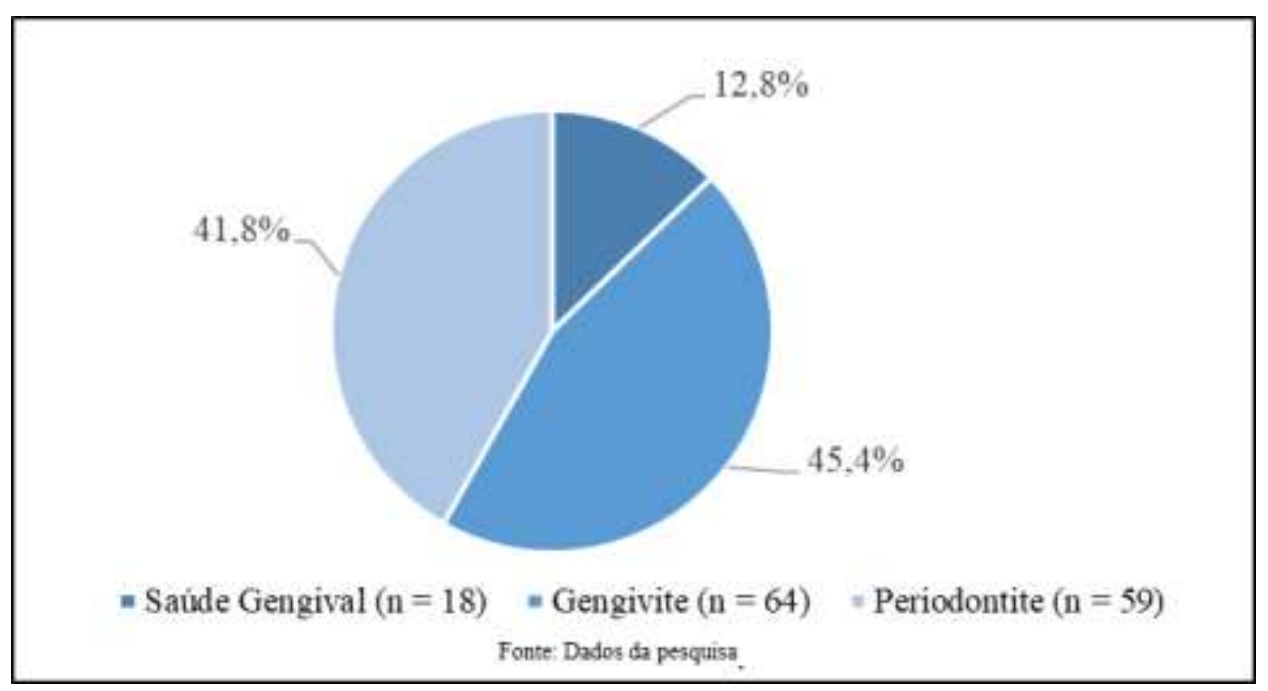

Em geral, as ocorrências de periodontite são caracterizadas por estágio II 28 (47,5\%), enquanto da gengivite são por generalizada $46(71,9 \%)$, conforme mostra a Tabela 2. 
Tabela 2. Estágios periodontite e gengivite.

VARIÁVEIS
ESTÁGIO PERIODONTITE $(\mathbf{N}=\mathbf{5 9})$
I
II
III
ESTÁGIO DA GENGIVITE $(\mathbf{N}=\mathbf{6 4})$
INDUZIDA POR BIOFILME
GENERALIZADA
Fonte: Dados da pesquisa.

Com os resultados contidos no Gráfico 2, é possível inferir que maiores valores de idade estão associados de maneira significante à existência de doença periodontal $(\mathrm{p}=0,008)$. Ressaltando que indivíduos com periodontite apresentaram média de idade 39,83 $( \pm 9,65)$ anos e para gengivite média de 35,80 ( $\pm 10,90)$ anos. Indivíduos com saúde gengival possuem média de idade centrada em $30,33( \pm 5,84)$ anos.

Gráfico 2. Distribuição da idade por diagnóstico da amostra.

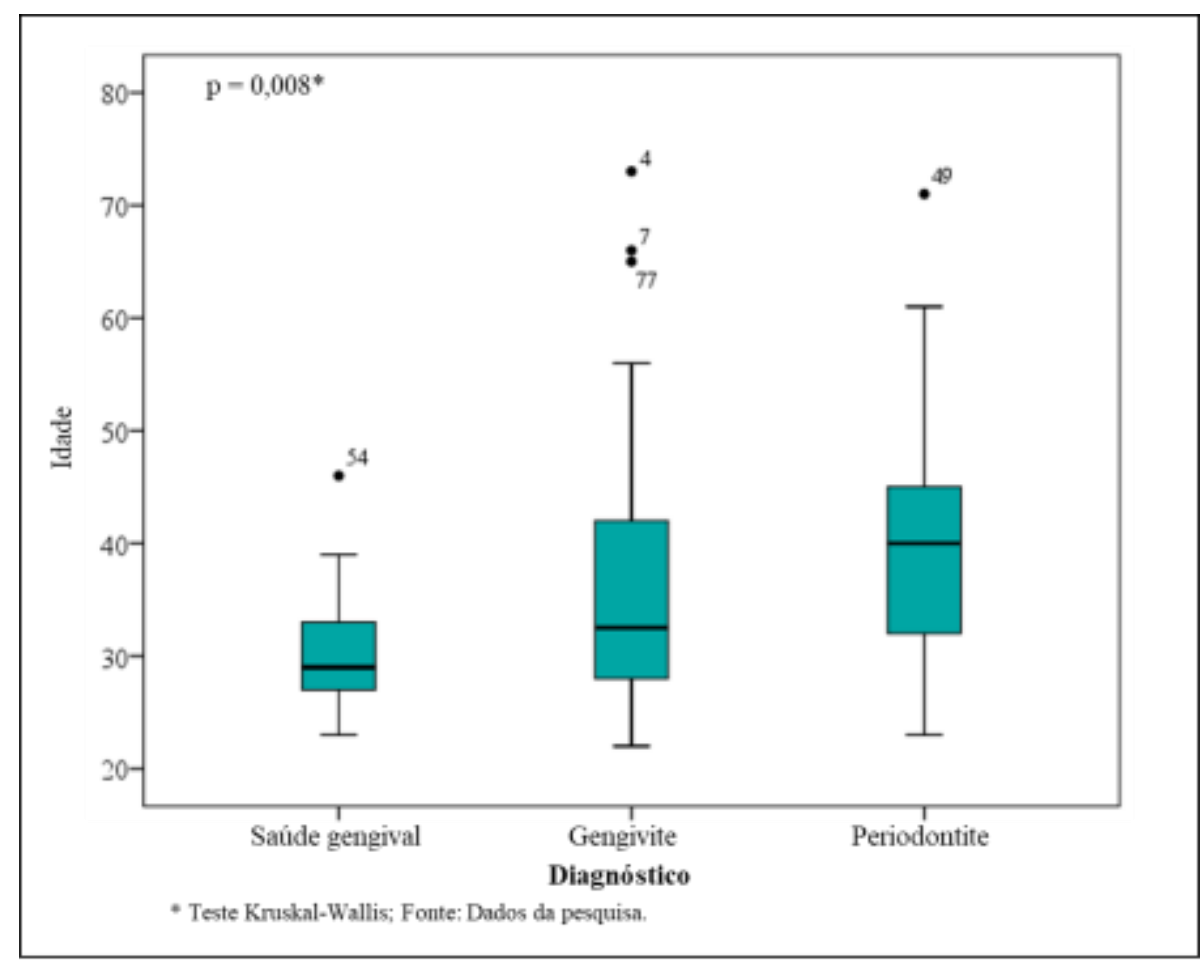

A ocorrência do diagnóstico entre os sexos se mostrou distribuída uniformemente, não sendo verificada correlação estatística significante $(\mathrm{p}=0,931)$. Em contrapartida, a distribuição dos casos de diagnóstico pela presença de doenças sistêmicas está correlacionada de maneira significante $(\mathrm{p}=0,019)$, mostrando que os casos de saúde gengival ocorrem mais em pessoas saudáveis, enquanto a presença de algum tipo de doença sistêmica implica em gengivite 41 (64,0\%) e periodontite 36 $(61,1)$, como mostra a Tabela 3. 
Tabela 3. Referência cruzada entre diagnóstico, sexo e doenças sistêmicas da amostra.

\begin{tabular}{|c|c|c|c|c|c|c|}
\hline \multicolumn{2}{|c|}{ VARIÁVEIS } & \multicolumn{3}{|c|}{ Diagnóstico, $n(\%)$} & & \multirow[t]{2}{*}{$p^{1}$} \\
\hline & & Saúde Gengival & Gengivite & Periodontite & & \\
\hline \multirow[t]{2}{*}{ SEXO } & Feminino & $10(55,6)$ & $34(53,2)$ & $30(50,8)$ & \multirow[b]{2}{*}{1} & 0,93 \\
\hline & Masculino & $8(44,4)$ & $30(46,8)$ & $29(49,2)$ & & \\
\hline \multirow{2}{*}{$\begin{array}{l}\text { DOENÇAS } \\
\text { SISTÊMICAS }\end{array}$} & Ausentes & $13(72,2)$ & $23(36)$ & $23(38,9)$ & \multirow[b]{2}{*}{9} & \multirow[t]{2}{*}{0,01} \\
\hline & Presentes & $5(27,8)$ & $41(64)$ & $36(61,1)$ & & \\
\hline
\end{tabular}

\section{Discussão}

O presente estudo avaliou a ocorrência das doenças periodontais fazendo analogia ao sexo, idade, raça, e presença de doenças sistêmicas. Para tanto, formam analisados 141 prontuários.

A idade dos indivíduos são fatores de risco mutáveis para presença ou agravo das doenças periodontais. Os dados da pesquisa evidenciaram maior incidência de doenças periodontais em idosos $(\mathrm{p}=0,008)$, corroborando com o estudo de Perrson, (2018, pp. 185-186) que relata ser possível o envelhecimento estar associado as condições periodontais e a doenças e complicações sistêmicas ou mesmo a fatores socioeconômicos. Rocha, Seibel, Nogueira e Rocha (2019, pp. 3-5) salientam que o processo inflamatório da doença periodontal em idosos está ligado as alterações celulares e teciduais, hábitos de higiene e a desregulação do sistema imunológico consequentes do envelhecimento. Da mesma forma, Hajishengallis (2014, pp. 30-37) afirma que alterações dependentes da idade na imunidade inata e no estado inflamatório podem aumentar a suscetibilidade à periodontite.

Apesar de haver uma predominância de mulheres na amostra - 74 (52,5\%), o sexo não exerceu influência sobre o diagnóstico periodontal, mostrando-se distribuído uniformemente, não sendo verificada correlação estatística significante $(\mathrm{p}=$ 0,931). Já Teixeira, et al. (2019, pp. 6-8), em um estudo transversal no período de 2014 a 2016, observaram que a incidência de doenças periodontais era maior em homens $(p<0,05)$. No entanto, para Oppermann, Haas, Rösing e Susin (2014, pp. 16-28) ainda não há clareza sobre a influência do sexo em relação a DP, acreditando que isso pode estar mais relacionado a fatores comportamentais do que genéticos.

Indivíduos leucoderma e melanoderma, não apresentaram relevância quanto a influência da raça em detrimento as doenças periodontais, ambas com 69 (48,9\%) dos casos. Em contrapartida, um estudo feito por Peres, Antunes, Boing, Peres e Bastos (2007, pp. 198-200), apontam para uma maior ocorrência de DP entre os negros, apresentando uma probabilidade 59\% maior.

Neste estudo, a doença periodontal mais prevalente foi a gengivite generalizada $(45,4 \%)$, seguida da peridontite estágio II (41, 8\%), achado considerado de grande relevância, uma vez que concorda com a literatura no que diz respeito a evolução da DP. Curtis, Diaz e Van Dyke (2020, pp. 21-23) explicam que o curso da DP progride da saúde periodontal para a gengivite e que, quando não tratada, pode evoluir para um quadro de perda de inserção como consequência da periodontite. No entanto, Wellapuli e Ekanayake (2017, pp. 155-156), ressaltam que a prevalência das doenças periodontais varia de acordo com o tipo da população.

Os indivíduos portadores de doenças sistêmicas estão correlacionados de maneira significante (Qui-quadrado, p = 0,019), em que os casos de saúde gengival ocorrem mais em pessoas saudáveis, contrapondo ao fato de que a presença de algum tipo de doença sistêmica implica em algum subtipo de doença periodontal, sendo a gengivite com $64,0 \%$ e a 
periodontite com 61,1\%. Lamster e Pagan, (2017b, pp. 67-74) sugerem que a inflamação periodontal desencadeia um estado inflamatório sistêmico e que, por isso, pode estar relacionada a doenças cardiovasculares, diabetes, problemas renais e sanguíneos.

De acordo com a pesquisa, pacientes portadores de problemas cardiovasculares, como a hipertensão, apresentaram sintomas característicos da periodontite. Alterações na microcirculação associadas à hipertensão podem causar isquemia no periodonto favorecendo o desenvolvimento da doença periodontal (Macedo Paizan \& Vilele-Martin, 2014). CarrizalesSepúlveda, Ordaz-Farías, Vera-Pineda e Flores-Ramírez (2018, pp. 2-6) explicam que por se tratar de uma doença multifatorial, a doença periodontal apresenta alterações sistêmicas como as relacionadas ao sistema cardiovascular e estão diretamente relacionadas à progressão da doença, além de ocorrer uma interrelação entre inflamação sistêmica e DPs, principalmente quando já estabelecida a periodontite, concordando com Sanz et al. (2020, pp. 3-11).

Nessa pesquisa, 5\% da amostra foi composta de pacientes com doenças renais. Miyata et al. (2019, pp. 14-15) evidenciaram que as periodontites afetam a resposta inflamatória de pacientes em diálise. De acordo com Kitamura et al. (2019, pp 5-19) a severidade da doença periodontal está associada de forma significativa no agravo das condições orais de pacientes em tratamento de hemodiálise ou transplantados.

Oliveira e Barbosa (2020, pp. 2-4) afirmam existir uma correlação entre doença periodontal e diabete mellitus, proporcionando maior risco de desenvolvimento ou progressão da periodontite, causando rápida destruição da inserção óssea alveolar, e, quando não tratada, pode agravar o controle metabólico do diabético. Para Albandar, Susin e Hughes (2018, pp. 172-184) o diabetes mellitus é a principal doença sistêmica que afeta a periodontite. Há indícios que revelam que a inflamação periodontal pode contribuir para o aparecimento e persistência da hiperglicemia. Neste estudo, indivíduos com diabetes compõe $15,6 \%$ da amostra.

Diante da alta ocorrência de doenças periodontais e gengivais na população estudada, faz-se necessário, investigar mais a fundo a quais fatores e preditores de risco estes indivíduos estão expostos. Além disso, esse estudo identificou a necessidade de campanhas de conscientização, prevenção, diagnóstico e tratamento adequado em âmbito social e multiprofissional relacionados a importância da manutenção da saúde periodontal como fator primordial para diminuição e melhor controle dos achados deste estudo.

\section{Conclusão}

Os dados avaliados a partir o perfil epidemiológico de pacientes atendidos em uma clínica escola evidenciou um alto índice da ocorrência de doenças periodontais, identificando a mais prevalente, pontuando os fatores de risco e correlacionandoas com doenças sistêmicas. Diante do exposto, observa-se a necessidade de mais estudos acerca do tema, além da criação de programas de prevenção e promoção da saúde bucal, visando, principalmente a qualidade de vida da população.

\section{Referências}

Albandar, J. M., Susin, C. \& Hughes, F. J. (2018). Manifestações de doenças sistêmicas e condições que afetam o aparelho de inserção periodontal: definições de casos e considerações diagnósticas. Journal of Clinical Periodontology, 45 (20), S171 - S189. https://doi.org/10.1111/jcpe.12947

Carrizales-Sepúlveda, E. F., Ordaz-Farías, A., Vera-Pineda, R., \& Flores-Ramírez, R. (2018). Periodontal Disease, Systemic Inflammation and the Risk of Cardiovascular Disease. Heart, Lung and Circulation, 27(11), 1327-1334. https://doi.org/10.1016/j.hlc.2018.05.102

Curtis, M. A., Diaz, P. I., \& Van Dyke, T. E. (2020b). The role of the microbiota in periodontal disease. Periodontology 2000, 83(1), 14-25. https://doi.org/10.1111/prd.12296

Estrela, C. (2018). Metodologia Científica: Ciência, Ensino, Pesquisa. Editora Artes Médicas

Hajishengallis G. Aging and its impact on innate immunity and inflammation: implications for periodontitis. $J$ Oral Biosci. 56:30-37. 10.1016/j.job.2013.09.001. 
Kinane, D. F., Stathopoulou, P. G., \& Papapanou, P. N. (2017). Periodontal diseases. Nature Reviews Disease Primers, 3(1). https://doi.org/10.1038/nrdp.2017.38

Kitamura, M., Mochizuki, Y., Miyata, Y., Obata, Y., Mitsunari, K., Matsuo, T., Sakai, H. (2019). Características patológicas da doença periodontal em pacientes com doença renal crônica e transplante renal. International Journal of Molecular Sciences, 20 (14), 3413. 10.3390 / ijms20143413

Lamster, I. B., \& Pagan, M. (2017b). Periodontal disease and the metabolic syndrome. International Dental Journal, 67(2), 67-77. https://doi.org/10.1111/idj.12264

Macedo Paizan ML, Vilele-Martin JF. Is there an association between periodontits and hypertension? Curr Cardiol Rev. 10:355-361. $10.2174 / 1573403 X 10666140416094901$.

Miyamoto, T., Kumagai, T., Khan, S., \& Reddy, M. S. (2019). Application of 2017 New Classification of Periodontal Diseases and Conditions to Localized Aggressive Periodontitis: Case Series. Clinical Advances in Periodontics, 9(4), 185-191. https://doi.org/10.1002/cap.10068

Miyata, Y., Obata, Y., Mochizuki, Y., Kitamura, M., Mitsunari, K., Matsuo, T., Ohba, K., Mukae, H., Nishino, T., Yoshimura, A., \& Sakai, H. (2019). Periodontal Disease in Patients Receiving Dialysis. International Journal of Molecular Sciences, 20(15), 3805. https://doi.org/10.3390/ijms20153805

Nazir, M. A. (2017). Prevalence of periodontal disease, its association with systemic diseases and prevention. Rev. International journal of health sciences, 11, (2), 72. https://www.ncbi.nlm.nih.gov/pmc/articles/PMC5426403.

Oliveira, L. M. L. \& Barbosa, L. M. (2020). Periodontitis and type 2 diabetes: critical review. RGO, Rev Gaúch Odontol. 68(2), 1-6. http://dx.doi.org/10.1590/1981-863720200005920190060

Oppermann, R. V., Haas, A. N., Rösing, C. K., \& Susin, C. (2014). Epidemiology of periodontal diseases in adults from Latin America. Periodontology 2000, 67(1), 13-33. https://doi.org/10.1111/prd.12061

Persson G. R. (2018). Periodontal complications with age. Periodontology 2000, 78(1), 185-194. https://doi.org/10.1111/prd.12227

Peres, M. A., Antunes, J. L. F., Boing, A. F., Peres, K. G., \& Bastos, J. L. D. (2007). Skin colour is associated with periodontal disease in Brazilian adults: a population-based oral health survey. Journal of Clinical Periodontology, 34(3), 196-201. https://doi.org/10.1111/j.1600-051x.2006.01043.x

Rocha, E. F. d., Seibel, A. B., Nogueira, A. N., \& Rocha, V. C. F. d. (2019). Envelhecimento humano e desenvolvimento da doença periodontal. Revista Eletrônica Acervo Saúde, (26), Artigo e775. https://doi.org/10.25248/reas.e775.2019

Rodrigues, K. T., Medeiros, L. A. D. M. D., Sousa, J. N. L. D., Sampaio, G. A. D. M., \& Rodrigues, R. D. Q. F. (2020). Associação entre condições sistêmicas e gravidade da doença periodontal em pacientes atendidos na Clínica-Escola da UFCG. Revista de Odontologia da UNESP, 49. https://doi.org/10.1590/18072577.02520

Santos, L. J. S., Bittencourt, M. S. P., Terezan, M. L. F., Rocha, L. F. M. D. (2020). Fluido gengival e atividade de doença periodontal. Revista Periodontia, 30, 189-194. https://pesquisa.bvsalud.org/portal/resource/pt/biblio-1128987?src=similardocs

Sanz, M., Marco Del Castillo, A., Jepsen, S., Gonzalez-Juanatey, J. R., D'Aiuto, F., Bouchard, P., Chapple, I., Dietrich, T., Gotsman, I., Graziani, F., Herrera, D., Loos, B., Madianos, P., Michel, J. B., Perel, P., Pieske, B., Shapira, L., Shechter, M., Tonetti, M., Vlachopoulos, C., \& Wimmer, G. (2020). Periodontitis and cardiovascular diseases: Consensus report. Journal of clinical periodontology, 47(3), 268-288. https://doi.org/10.1111/jcpe.13189

Silva, G. C. B. d., Melo Neto, O. d. M., Nascimento, A. M. V. d., Santos, C. A. O. d., Nóbrega, W. F. S., \& Souza, S. L. X. d. (2020). História Natural da Doença Periodontal: uma revisão sistematizada. Research, Society and Development, 9(7), Artigo e607974562. https://doi.org/10.33448/rsd-v9i7.4562

Slots, J. (2020). Primer on etiology and treatment of progressive/severe periodontitis: A systemic health perspective. Periodontology 2000, 83(1), 272-276. https://doi.org/10.1111/prd.12325

Teixeira, F. C. F., Marín-León, L., Gomes, E. P., Pedrão, A. M. N., Pereira, A. D. C., \& Francisco, P. M. S. B. (2019). Perda de inserção periodontal e associações com indicadores de risco sociodemográficos e comportamentais. Revista de Odontologia da UNESP, 48. https://doi.org/10.1590/1807-2577.09519

Wellapuli, N., \& Ekanayake, L. (2017). Prevalence, severity and extent of chronic periodontitis among Sri Lankan adults. Community dental health, 34(3), 152-156. https://doi.org/10.1922/CDH_4070Wellapuli05 